\title{
Validación del constructo, contenido y confiabilidad de indicadores de proceso para la evaluación de la dimensión curricular en la educación superior: el caso del Instituto Tecnológico de Costa Rica
}

\author{
Validation of construct, content and reliability of \\ process-type indicators used for the assessment of \\ curricular process dimension at higher education: \\ the case of Costa Rica Institute of Technology
}

Rosa Inés Lira-Valdivia'

Fecha de recepción: 31 de julio del 2013 Fecha de aprobación: 30 de noviembre del 2013

Lira-Valdivia, R. Validación del constructo, contenido y confiabilidad de indicadores de proceso para la evaluación de la dimensión curricular en la educación superior: el caso del Instituto Tecnológico de Costa Rica. Tecnología en

Marcha.Vol. 27, № 2. Pág II7-136

Doctora en Intervención educativa por la Universidad de Valencia, España. Máster en Planificación Curricular por la Universidad de Costa Rica. Licenciada en Administración Educativa con énfasis en currículo por la Universidad de Costa Rica. Asesora Académica. Centro de Desarrollo Académico (CEDA). Instituto Tecnológico de Costa Rica. Costa Rica. Correo electrónico: rlira@itcr.ac.cr. 


\section{Palabras clave}

Indicadores de proceso; indicadores de calidad; proceso curricular; diseño curricular; análisis de contenido; evaluación curricular; evaluación institucional.

\section{Resumen}

En este trabajo se presentan los resultados de la validación efectuada a un conjunto de indicadores con el propósito de utilizarlos en la evaluación de la dimensión Proceso Curricular en una instancia de educación superior, en este caso el Instituto Tecnológico de Costa Rica. Se muestran los resultados relativos a los tres tipos de validez: de constructo, contenido y confiabilidad, incluyendo las correspondientes conclusiones del estudio. El trabajo se enmarca en una investigación evaluativa de enfoque cuantitativo de corte doctoral con la Universidad de Valencia y el Instituto Tecnológico de Costa Rica, utilizando como metodología el juicio de expertos.

Se concluye que los resultados de validez alcanzados ofrecen evidencia suficiente para respaldar los indicadores como ítems de calidad, ampliamente confiables para proporcionar información válida en la evaluación de la dimensión Proceso Curricular. Asimismo, se estima que estos indicadores proveerán información pertinente y serán un insumo importante para la toma de decisiones sobre políticas curriculares y evaluativas en la educación superior.

\section{Key words}

Curricular process; curricular assessment; processtype indicators; curriculum design; content analysis; institutional evaluation; assessment items for academic development; higher education.

\begin{abstract}
This paper is a part of a dissertation carried out as a doctoral study with the University of Valencia, to design Process-type-items for the assessment of Curriculum Process Dimension in Higher Education. Methodologically these indicators have been validated by a research evaluation based on expert judgment using a quantitative approach. This series of indicators has been built for Costa Rica Institute of Technology. The outcomes of three types of validity: construct, content and reliability are shown in this article. It is considered that the results achieved, provide sufficient evidence to support the proposed Process-type Indicators, as widely trusted and as quality items to provide valid and reliable information in assessing Curriculum Process Dimension. These indicators will also provide relevant data for making educational policy decisions in the area of curriculum development.
\end{abstract}

\section{Introducción}

Una de las formas de conocer la eficiencia y la calidad de un sistema educativo es a través de los datos cuantitativos que brindan los indicadores. Por la información que proveen y para las audiencias evaluativas, los indicadores constituyen un mecanismo fundamental en procesos de valoración y son además los insumos adecuados para determinar la calidad educativa de los escenarios formativos universitarios. En el ámbito curricular, la preocupación de las universidades por ser garantes de la calidad de sus ofertas educativas ha hecho que la evaluación del área curricular se convierta en uno de los elementos importantes de una reforma educativa. Esto implica que las universidades deben tener la posibilidad y la capacidad de contar con instrumentos confiables para llevar a cabo la valoración de la dimensión curricular.

Es en este marco que se ubica este artículo, cuyo objetivo principal es proponer una serie de indicadores para la evaluación de la dimensión denominada en este estudio Proceso Curricular y, al mismo tiempo, mostrar los resultados obtenidos en la validación a la que se sometieron dichos indicadores. No obstante, es importante ubicar este escrito en el marco general de la investigación doctoral que le 
da origen. Es decir, la dimensión que aquí se muestra es una de las cinco que se han conformado para constituir el área de Formación Universitaria, como parte de un planteamiento global que tiene como finalidad formular una propuesta de evaluación institucional para el Instituto Tecnológico de Costa Rica (ITCR). En el diseño madre, Formación Universitaria está constituida por las siguientes dimensiones: Proceso Curricular, Proceso de Enseñanza, Proceso Aprendizaje, Proceso Evaluación de Aprendizajes y Proceso Investigación. Formación Universitaria, a su vez, es una de las cinco áreas utilizadas y para las cuales se crearon indicadores de proceso y de producto y así poder fundamentar el planteamiento de evaluación institucional de la propuesta mencionada.

Por su extensión, en este artículo se presentan solamente los resultados obtenidos en relación con el juzgamiento de los indicadores de proceso en torno a la validez de constructo, contenido y confiabilidad de los reactivos que componen la dimensión Proceso Curricular. La validación respectiva se ha hecho mediante juicio de expertos con jueces nacionales e internacionales. La pertinencia de este artículo apunta no solo a la necesidad de proponer ítems que sean referentes claros, válidos y confiables sino también que permitan, mediante su aplicación, recabar y obtener información sobre el estado de uno de los procesos formativos, en este caso, del proceso curricular. Asimismo, estos indicadores recopilarán evidencia suficiente para la toma de decisiones sobre políticas evaluativas curriculares en torno al desarrollo curricular del área de Formación Universitaria.

\section{Objetivos}

a) Construir un conjunto de indicadores de proceso que sean válidos y confiables para evaluar la dimensión Proceso Curricular como componente del área de Formación Universitaria. b) Validar el conjunto de indicadores tipo proceso mediante los respectivos análisis de validez de contenido, validez de constructo y de confiabilidad.

\section{Marco teórico conceptual}

La historia del currículo marca un trayecto complejo y diverso que, según Penalva (2007, citado por Lira, 2010, p. 8) "no proviene de una disciplina académica, ni de una línea de investigación única y monolítica, sino, más bien, de una corriente educativa muy diversa". Lo anterior significa que históricamente el currículo ha influido y se ha dejado influir por una gran variedad de teorías y tendencias pedagógicas y culturales. Cada una de ellas representa fehacientemente tratados de pensamiento curricular, planteamientos y fundamentaciones teóricas, aproximaciones teórico-contextuales, sociales e históricas acerca del currículo, cuya trascendencia continúa impactando los procesos de enseñanza y aprendizaje de los sistemas educativos y universitarios en general.

Autores como Follari y Berruezo (1981), Díaz Barriga (1984), De la Torre (1999), Carr y Kemmis (1988), De Alba (1994) o Coll (1988) han señalado la urgente necesidad de una transformación en la conformación de los currícula. Han evidenciado el gran vacío de espacios para el análisis y actualización de temas curriculares atinentes al diseño de las carreras en las universidades. En tales circunstancias, el marco teórico conceptual de referencia curricular que sustenta el enfoque teórico de este artículo se ubica en la perspectiva de autores como Grundy (1987), Posner (2000), Stenhouse (1991) y Flores Ochoa (2000), de quienes se han analizado sus axiomas para conceptualizar los principales componentes que conforman la dimensión Proceso Curricular en el marco de esta investigación.

Con base en lo anterior, se entiende por dimensión Proceso Curricular la siguiente conceptualización: "Es una construcción cultural que busca la concreción específica de una teoría pedagógica para volverla efectiva y asegurar el aprendizaje y el desarrollo de un grupo particular de alumnos según la cultura, época y comunidad de las cuales forma parte" (Lira, 2013, p. 176). Para los efectos específicos de este estudio, dicha dimensión se operacionaliza en dos grandes categorías: Diseño curricular' y Carrera².

I Diseño curricular: En este artículo se entiende diseño curricular de la siguiente manera: "Es el componente vital del proceso curricular, que consiste en un transcurso integrado de construcción social, el cual identifica el conjunto de saberes que mejor actúa en la complejidad del diseño, con el fin de asegurar los conocimientos, habilidades y facultades que conformarán el plan de formación de los futuros profesionales de una carrera universitaria. Así, el diseño curricular es el encargado de operativizar el proceso enseñanza y aprendizaje en el plan de formación integral, de tal manera que ese diseño curricular sea una oferta académica auténtica, valiosa, no solo para los efectos de formación profesional universitaria, sino también útil y funcional para afrontar y resolver situaciones nuevas propias de la profesión" (Lira, 2010, pp. 28-29).

2 El vocablo Carrera como categoría operativa en el marco de este artículo está conformado por los siguientes componentes que son 
De igual manera, ambas subdimensiones han sido operacionalmente precisadas mediante componentes y estos, a su vez, se operativizan en indicadores de tipo proceso. Dichos elementos son, en el caso de la categoría Diseño curricular, los siguientes: Principios curriculares, Perfil, Estructura curricular y Elementos normativos institucionales. Con relación a la segunda subdimensión, los componentes que la constituyen son: Autorregulación, Tutorías estudiantiles, Infraestructura, Seguimiento e Idoneidad.

Es importante mencionar que para lograr un acercamiento a la realidad empírica de estos componentes, se aplica lo indicado por Lazarfeld (1985, citado por Rodríguez J., 2000, p. I5 I) en relación con lo importante que resulta la "elaboración de indicadores para la cuantificación de las dimensiones descritas". Es así como, desde la perspectiva instrumental evaluativa, se construyeron indicadores de tipo proceso para valorar los respectivos componentes, los cuales se conceptualizan para los efectos de la investigación de la siguiente manera: "los indicadores de proceso son los motores impulsores, los medios y las acciones específicas que pretenden generar los productos esperados y lograr los fines y resultados propuestos" (Lira, 2013, p. 122). Asimismo, para el logro de los objetivos y la respectiva validación de los indicadores de proceso, se utilizaron los siguientes dos criterios: relevancia y susceptibilidad de mejora, cada uno de los cuales tiene su respectiva conceptualización, por ejemplo, relevancia es definido así: "El indicador es relevante cuanto mejor represente al objeto o subdimensión con la cual se relaciona" (Lira, 2013, p. 144). En relación con el criterio de susceptibilidad de mejora, dicha conceptualización se refiere a "La posibilidad de mejoramiento para una condición predeterminada que expresa el indicador, siempre y cuando esa posibilidad de mejora esté sustentada en datos o procesos viables, a partir de los cuales se pueda variar la condición de ese objeto determinado y pueda ser ejecutado con los recursos propios que posea la institución (humanos, fisicos, económicos) para realizar dicha mejora" (Lira, 20 I3, p. I44).

Aunado a lo anterior, el marco empírico evaluativo sobre el cual se fundamenta este artículo hace men-

indispensables para promover el desarrollo intelectual y formal de los estudiantes durante su proceso de formación universitaria: autorregulación, tutorías estudiantiles, infraestructura, seguimiento de estudiantes y profesores e idoneidad docente (Lira, 20I3, p. 213). ción de los conceptos utilizados a fin de operativizar las respectivas validaciones a la que se sometieron los susodichos indicadores. Es así como la validez de contenido se define a la luz de dos autores: Krippendorff (1997, p. 81), quien la define como el "conjunto de métodos y técnicas de investigación destinados a facilitar la descripción e interpretación sistemática de los componentes semánticos y formales de todo tipo de escritura o mensaje y la formulación de inferencias válidas acerca de los datos reunidos". De igual manera, Hernández, Fernández y Baptista (2003, p. 347) la entienden así: "es el grado en el que la medición representa al concepto medido". Por su parte, la definición de validez de constructo que ampara esta investigación se basa en autores como Greeno (1980), Snow (1980), Seller (1988) y Leyva (200 I, citados por Lira, 20 I3, p. I 50), quienes señalan varios pasos para establecer la validez de constructo, entre los cuales están: "Elegir o construir una teoría para la definición de conceptos y la determinación a priori de las relaciones entre ellos. Seleccionar indicadores que representen cada uno de los conceptos contenidos en la teoría. La constatación de estos dos pasos requiere de análisis lógicos a través de jueces expertos". Para McMillan \& Schumacher (2005, p. 633), este concepto "es el tipo de validez externa que se refiere al grado en que el estudio representa el constructo fundamental". En relación con la fiabilidad, esta se entiende como "el grado en que son consistentes las medidas de un test" (McMillan \& Schumacher, 2005, p. 622). La aplicación de estos conceptos se explica en el apartado de análisis realizados. Los anteriores conceptos constituyen los principales referenciales teóricos curriculares y los empíricos evaluativos sobre los cuales se desarrolla el presente artículo.

\section{Metodología}

Con respecto al tipo de indagación efectuada, la presente es una investigación evaluativa basada en el juicio de expertos y utiliza un enfoque cuantitativo. En función del análisis y las técnicas utilizadas para la recopilación de la información, se clasifica como una investigación descriptiva y exploratoria. Es de tipo descriptivo porque pretende caracterizar y extraer conclusiones sobre "dimensiones y componentes del fenómeno a investigar" (Hernández, Fernández y Baptista, 2003, p. I 17); en este caso en particular se busca extraer conclusiones sobre la dimensión 
denominada Proceso curricular. Es exploratoria, ya que pretende indagar acerca de las características de las distribuciones de las variables y busca "identificar relaciones entre variables" (Hernández et ál., 2003, p. I 17); en nuestro caso, indaga en el comportamiento de las variables de proceso involucradas a la luz del criterio de expertos distinguiendo, mediante ello, los indicadores promisorios de los que no lo son, en función de la dimensión y de los procesos que representan; además "evalúa una práctica específica en un lugar determinado" (McMillan \& Schumacher, 2005, p. I |6).

\section{Muestra}

Se trabaja con el concepto de "grupo poblacional" entendido como el "subconjunto de sujetos que tienen las características de la población, pero que no son representativos en cuanto al número para poder establecer generalizaciones" (Jornet y Suárez, 2008b, p. 9). El objetivo de trabajar con grupos finitos de la población, según lo indican estos mismos autores, es "poder estudiar la viabilidad de posibles hipótesis - analizar la adecuación de instrumentos de medida durante su elaboración" (Jornet y Suárez, 2008b, p .5).

En este caso específico, se está estudiando la viabilidad de los componentes y se analiza cuán adecuados son los diferentes indicadores que conforman la dimensión Proceso curricular. De igual manera, interesa hacer inferencias sobre los ítems construidos, una vez sometidos a procesos de análisis estadísticos, para determinar cuáles de ellos son más susceptibles de generar mejora en los procesos y en las dimensiones que hemos identificado como tales y cuál es la relevancia que ostentan en esos procesos. Por tanto, la muestra con la que se trabaja no es probabilística o aleatoria sino más bien intencional y corresponde a lo que Elorza (2000, citado por Torres, 2003, p. 57) determina como tipo de "muestreo por conveniencia pues se procede a ponerse en contacto con unidades de muestreo apropiadas". Sirva además lo indicado por Hernández, Fernández y Batista (2003, p. 327) para reforzar lo anteriormente manifestado: "la ventaja de una muestra no probabilística es su utilidad para determinado diseño de estudio que requiere no tanto una 'representatividad' de elementos de una población, sino una cuidadosa y controlada elección de sujetos con ciertas características". De ahí se deriva la utilidad y la necesidad de trabajar en esta investigación con un grupo de expertos que, dadas sus cualidades, experiencias y vivencias en el campo de la evaluación, interesa más la profundidad y la calidad de la información aportada más que la cantidad o la estandarización.

\section{Sujetos}

En virtud de lo anterior, se trabajó con dos grupos de expertos: uno a lo interno del ITCR y el otro a lo externo con expertos de los ámbitos nacional e internacional, utilizando como criterios de selección los siguientes: a. Comité interno: académicos universitarios del ITCR con experiencia en la dirección o ejecución de procesos de autoevaluación, académicos con experiencia y participación en procesos de certificación externa de programas o carreras universitarias; b. Comité externo: académicos universitarios con experiencia en evaluación de programas y carreras universitarias; académicos que trabajen en o para organismos 0 instancias relacionadas con procesos de evaluación institucional, certificación de calidad externa o procesos de acreditación nacional e internacional de universidades. El grupo poblacional inicial fue de 37 expertos y el final fue de 28 especialistas. Estos académicos provienen de universidades nacionales costarricenses y de universidades españolas, colombianas, panameñas, venezolanas y chilenas (ver figura I).

\section{Análisis realizados}

Para el tratamiento y análisis de los datos se utiliza el paquete estadístico SPSS versión I8, para el procesamiento de datos el programa Microsoft Excel para Windows 7. Como se mencionó anteriormente, este artículo forma parte de un trabajo más amplio de investigación en la cual se realizaron análisis pertenecientes a la estadística descriptiva y a la inferencial. Así por ejemplo, para establecer la calidad técnica del instrumento y de los ítems construidos, se realizaron los siguientes análisis: nivel de dificultad de la prueba, poder de discriminación, intensidad del coeficiente de homogeneidad de la prueba alfa de Cronbach y alfa total, coeficiente de validez de los ítems, análisis de matriz varianza/covarianza, matriz de interrelaciones, nivel de dificultad de los ítems e intensidad de la correlación. Asimismo, se efectuaron análisis descriptivos y de tendencia central, de congruencia entre jueces, de homogeneidad, de comprobación de supuestos, correlacionales y prueba $t$ para muestras independientes, estas últi- 


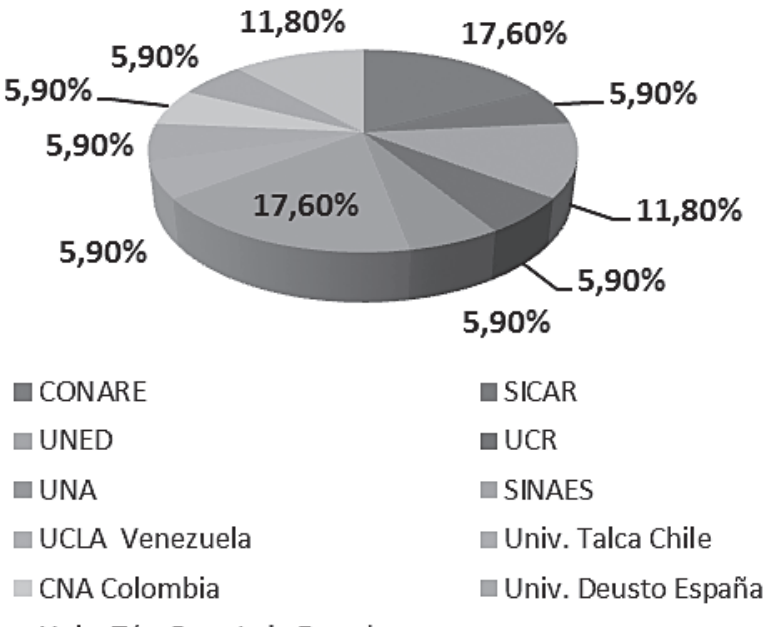

Figura I. Porcentaje de evaluadores del comité externo de validación agrupados según la institución a la que pertenecen.

mas pertenecientes a la estadística inferencial. Para los efectos particulares de este artículo, se detallan los análisis que se realizaron en torno a la validez de contenido, constructo y confiabilidad.

En relación con el análisis de fiabilidad de cada uno de los indicadores de proceso de la dimensión Curricular, se obtuvo el Alfa de Cronbach y se efectuó el análisis de consistencia interna mediante la obtención del estadístico correlación elemento total corregido. El análisis coeficiente de homogeneidad o correlación ítem-test se utilizó como medida de correlación entre la puntuación de cada indicador con la suma de las puntuaciones de los otros ítems de la escala. Este índice permitió conocer la contribución de cada indicador a la fiabilidad total de cada componente.

Para determinar la validez de constructo se aplica el análisis de conglomerados jerárquicos y el análisis de factores. El primero se utilizó con el fin de establecer grupos o tipologías semánticas entre los indicadores creados, a fin de descubrir agrupaciones naturales entre ellos lo más homogéneas a lo interno de éstas. Este hecho permitió hacer las inferencias del caso y relacionar los respectivos contenidos teóricos de los ítems con la dimensión y el constructo que están representando. El segundo, es decir, el análisis de factores, se utilizó para determinar la validez de constructo aplicando para ello el análisis de Componentes Principales (ACP) con rotación oblicua, matriz de correlaciones, índice de KMO y la prueba de Esfericidad de Bartlett.

En la determinación de la validez de contenido de los indicadores de proceso se aplican los siguientes análisis: el cociente de variación, media aritmética, rangos promedio y el análisis perfil de importancia de los indicadores respecto al contenido. Aplicando el significado de la validez de contenido y mediante estos análisis, se busca facilitar la elucidación sistemática de indicadores de proceso y conducir la interpretación de los jueces, mediante la determinación lo más exacta posible de las características y significados que estos ítems representan. Así, el estudio de las valoraciones individuales que dieron los expertos sobre cada uno de los indicadores y su sistematización se realizó utilizando para ello -como se mencionó anteriormente- el cociente de variación. Asimismo, se recurrió al análisis de media aritmética, el cual, por sí solo, es un indicador de la validez de contenido (Jornet y Suárez, 2008b, s.p). De esta manera, mediante la media y los rangos promedio se aplicó el estudio de Perfil de importancia de los indicadores respecto al contenido, posibilitando con ello la clasificación y determinación de categorías conceptuales en relación con el contenido de los reactivos evaluados como los "más relevantes y más susceptibles de mejora", "menos relevantes y menos susceptibles de mejora" y además aquellos que "no presentaron polaridades en sus respectivas evaluaciones" (ver cuadro 5). 


\section{Resultados}

Indicadores de proceso

En relación con la creación de los indicadores tipo proceso que conforman la dimensión Proceso curricular, estos se presentan en el cuadro I. Sin embargo, es importante ubicar este grupo de indicadores en el marco general de la investigación madre, en la cual solamente para el caso de la evaluación del área Formación Universitaria se construyeron 302 indicadores de proceso y se sometieron a la respectiva depuración; el figura 2 muestra el resumen de dicho análisis realizado al área de Formación Universitaria. El cuadro I muestra específicamente los ítems que atañen a la dimensión Proceso curricular, una vez realizada la depuración técnica del instrumento. En esa línea y en forma breve, algunos resultados obtenidos en relación con la calidad técnica de los indicadores construidos son los siguientes: la dificultad de los ítems corresponde a un nivel medio de dificultad o sea $\left(P_{i}>0.25\right.$ y $\left.\leq 0,75\right)$; el poder de discriminación de los indicadores se manifiesta en discriminación Máxima, incluyendo ítems modales o sea con puntuación $\left(\sigma^{2}=0,25\right)$; también se registró poder de discriminación Crítica, es decir, indicadores con puntajes $\left(\sigma^{2}=0,1-0,2\right)$. Con respecto al nivel de dificultad del instrumento utilizado, los resultados arrojan un dato de $C V=9,4 \%$, lo cual significa que dicho nivel es homogéneo.
Con respecto a los resultados logrados, los índices de fiabilidad obtenidos son altos en todos los componentes y las dos subdimensiones que forman la dimensión Proceso curricular. Los indicadores de proceso evaluados desde los criterios de relevancia y de la susceptibilidad de mejora y mediante la valoración tanto de los jueces del Comité interno como de los expertos del Comité externo son resultados satisfactorios, significando que aportan positivamente a la fiabilidad de los componentes que representan dichos indicadores y al constructo en general. Estos se presentan en la cuadro 2.

Resultados de análisis de conglomerados jerárquicos

En relación con estos resultados, el gráfico Dendrograma de Ward fue de gran utilidad para realizar dicho análisis. Se utilizó el método Ward con una medida de intervalo distancia euclídea al cuadrado conglomerando los indicadores por variables, en vez de por casos. Con este estudio se demuestra que la cercanía natural de los indicadores está conforme con las tipologías semánticas obtenidas en el análisis de contenidos que respaldan el significado de los constructos estudiados, corroborando de esta manera la validez de constructo de los indicadores tanto para el criterio de relevancia como para el de susceptibilidad de mejora en ambas áreas indicadas. Se presenta únicamente el Dendrograma que contiene los resultados referentes al primer

\section{Indicadores Proceso}

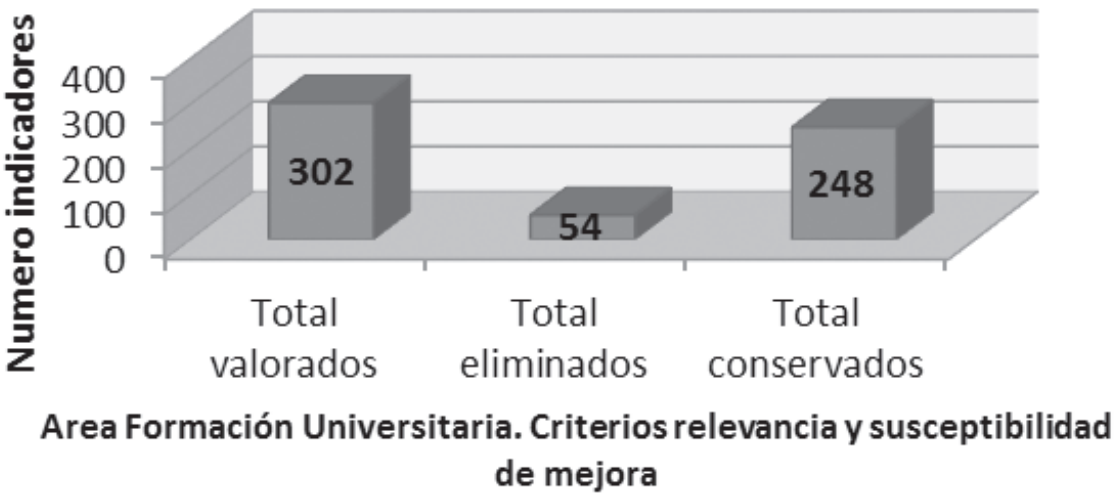

Figura 2. Resumen depuración de los indicadores de proceso del área de Formación Universitaria. Criterios relevancia y susceptibilidad de mejora. 
Cuadro I. Indicadores de proceso: Área Formación Universitaria. Dimensión Proceso Curricular.

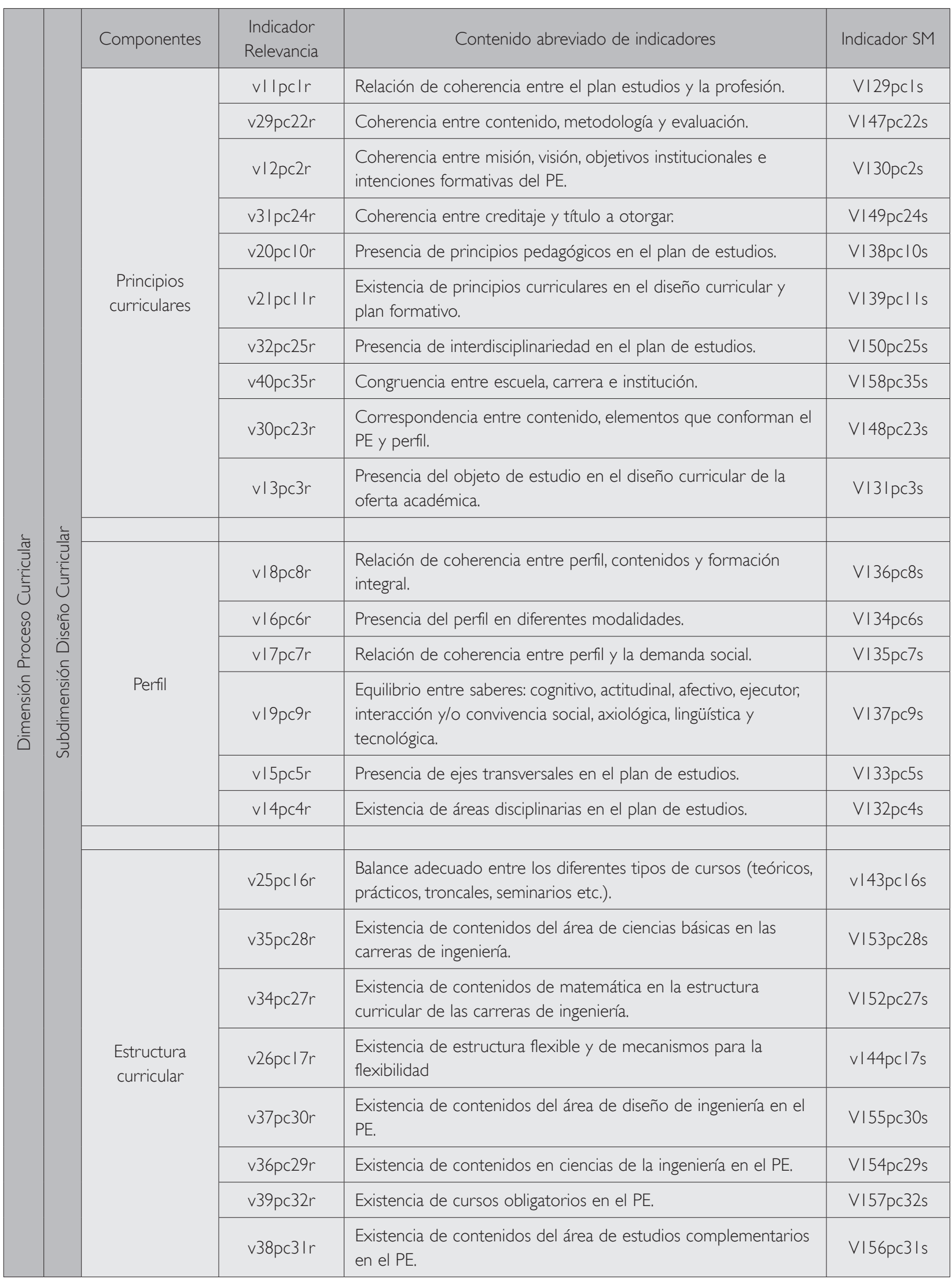


Continuación

\begin{tabular}{|c|c|c|c|c|}
\hline & Componentes & $\begin{array}{l}\text { Indicador } \\
\text { Relevancia }\end{array}$ & Contenido abreviado de indicadores & Indicador SM \\
\hline & \multirow{6}{*}{$\begin{array}{l}\text { Elementos } \\
\text { normativos } \\
\text { institucionales }\end{array}$} & v22pc|2r & El plan de estudios tiene objetivos claramente definidos. & VI40pcI2s \\
\hline & & $\mathrm{v} 23 \mathrm{pc} \mid 3 \mathrm{r}$ & $\begin{array}{l}\text { La fundamentación de la carrera conjuga la evolución del } \\
\text { conocimiento de la disciplina con el entorno social y nacional. }\end{array}$ & VI4Ipc|3s \\
\hline & & v24pc|4r & $\begin{array}{l}\text { EI DC presenta el elemento "justificación de la carrera" } \\
\text { elaborado con base en las necesidades del entorno social } \\
\text { nacional y en estudios de mercado. }\end{array}$ & VI42pc|4s \\
\hline & & v28pc20r & $\begin{array}{l}\text { El DC presenta número de actualizaciones de los contenidos y } \\
\text { de los conocimientos declarados en el plan de estudios. }\end{array}$ & VI46pc20s \\
\hline & & v27pc|8r & $\begin{array}{l}\text { Muestra evidencia constante de que el plan de estudios } \\
\text { promueve la formación integral del estudiante. }\end{array}$ & VI45pc|8s \\
\hline & & v33pc26r & $\begin{array}{l}\text { Todo DC presenta los programas oficiales que conforman el } \\
\text { plan de estudios. }\end{array}$ & VI4Ipc26s \\
\hline \multirow{12}{*}{ 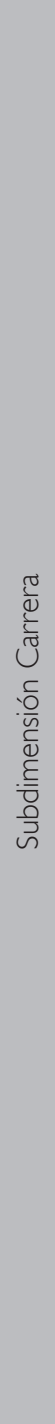 } & \multirow{6}{*}{ Autorregulación } & v42pc37r & $\begin{array}{l}\text { La carrera de ingeniería tiene mecanismos establecidos para la } \\
\text { actualización de saberes en el plan de estudios acorde con los } \\
\text { niveles de calidad señalados por organismos internacionales de } \\
\text { ingeniería. }\end{array}$ & VI60pc37s \\
\hline & & v4lpc36r & $\begin{array}{l}\text { Existencia de lineamientos escritos y planificados para la revisión } \\
\text { periódica del plan de estudios. }\end{array}$ & VI59pc36s \\
\hline & & v44pc40r & $\begin{array}{l}\text { Análisis de las acciones formativas en los cursos de la carrera } \\
\text { de ingeniería. }\end{array}$ & VI62pc40s \\
\hline & & v64pc68r & $\begin{array}{l}\text { Concienciación en profesores y alumnos en torno a la } \\
\text { autorregulación de la carrera. }\end{array}$ & VI82pc68s \\
\hline & & v43pc38r & $\begin{array}{l}\text { Participación de académicos en el diseño curricular de las } \\
\text { carreras. }\end{array}$ & VI6Ipc38s \\
\hline & & v59pc6/r & $\begin{array}{l}\text { La carrera de ingeniería demuestra liderazgo en la comunidad } \\
\text { académica mediante los aportes académicos e investigativos de } \\
\text { sus funcionarios. }\end{array}$ & VI77pc6Is \\
\hline & \multirow{4}{*}{$\begin{array}{l}\text { Tutorías } \\
\text { estudiantiles }\end{array}$} & v48pc46r & $\begin{array}{l}\text { La carrera presenta lineamientos normativos para la } \\
\text { presentación de trabajos académicos del estudiantado. }\end{array}$ & VI66pc46s \\
\hline & & v45pc42r & $\begin{array}{l}\text { La escuela realiza actividades de apoyo, tutoría y orientación } \\
\text { académica para el estudiantado. }\end{array}$ & V163pc42s \\
\hline & & v49pc47r & $\begin{array}{l}\text { Listado de eventos académicos y culturales organizados por la } \\
\text { carrera en la que participan los estudiantes. }\end{array}$ & V167pc47s \\
\hline & & v50pc48r & $\begin{array}{l}\text { Estrategias y mecanismos para incentivar la consulta bibliográfica } \\
\text { y el uso de las TICS. }\end{array}$ & VI68pc48s \\
\hline & \multirow[t]{2}{*}{ Infraestructura } & v46pc44r & $\begin{array}{l}\text { Tenencia de equipos especializados y suficientes para la } \\
\text { especialización de la disciplina. }\end{array}$ & VI64pc44s \\
\hline & & $v 47 p c 45 r$ & Tenencia de infraestructura adecuada. & VI65pc45s \\
\hline
\end{tabular}




\begin{tabular}{|c|c|c|c|c|}
\hline & Componentes & $\begin{array}{l}\text { Indicador } \\
\text { Relevancia }\end{array}$ & Contenido abreviado de indicadores & Indicador SM \\
\hline & & $v 54 p c 54 r$ & $\begin{array}{l}\text { Accesibilidad para el uso de laboratorios básicos y } \\
\text { especializados. }\end{array}$ & VI72pc54s \\
\hline & & $v 54 p c 54 r$ & $\begin{array}{l}\text { Accesibilidad para el uso de laboratorios básicos y } \\
\text { especializados. }\end{array}$ & VI72pc54s \\
\hline & & v56pc56r & $\begin{array}{l}\text { Tenencia suficiente de recursos informáticos acorde al } \\
\text { desarrollo tecnológico. }\end{array}$ & VI74pc56s \\
\hline & & v5 I pc50r & $\begin{array}{l}\text { Relación entre el número de estudiantes y la cantidad de } \\
\text { recursos informáticos. }\end{array}$ & VI69pc50s \\
\hline & & v55pc55r & $\begin{array}{l}\text { Tenencia de laboratorios y talleres especializados para el } \\
\text { desarrollo de competencias especializados. }\end{array}$ & VI73pc55s \\
\hline \multirow{9}{*}{ 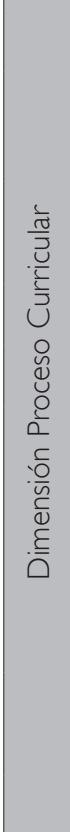 } & \multirow{4}{*}{ Seguimiento } & $v 52 p c 51 r$ & Base de datos sobre rendimiento estudiantil. & VI70pc5Is \\
\hline & & v57pc57r & $\begin{array}{l}\text { Listado de participación en actividades de desarrollo profesional } \\
\text { y pedagógico del profesorado. }\end{array}$ & VI75pc57s \\
\hline & & v58pc60r & $\begin{array}{l}\text { Presentación de proyectos para promover interacción con la } \\
\text { industria. }\end{array}$ & VI76pc60s \\
\hline & & v53pc52r & Mejoramiento metodológico en la enseñanza de la ingeniería. & VI7Ipc52s \\
\hline & \multirow{5}{*}{ Idoneidad } & v60pc63r & $\begin{array}{l}\text { Adecuado nivel académico que demuestra la idoneidad del } \\
\text { profesorado. }\end{array}$ & VI78pc63s \\
\hline & & v62pc65r & Listado de publicaciones científicas del profesorado. & VI80pc65s \\
\hline & & v65pc69r & $\begin{array}{l}\text { Listado de cursos sobre educación continua en el ámbito de } \\
\text { ingeniería para profesores y egresados. }\end{array}$ & VI83pc69s \\
\hline & & v6lpc64r & $\begin{array}{l}\text { Presenta evidencia referente a la experiencia en la industria por } \\
\text { parte del cuerpo docente del área de ingeniería. }\end{array}$ & VI79pc64s \\
\hline & & v63pc66r & Participación del profesorado en sociedades de aprendizaje. & VI8Ipc66s \\
\hline
\end{tabular}

componente, denominado Principios curriculares, dada su extensión.

Según el Dendrograma, el primer conglomerado está constituido por los elementos más cercanos o más similares entre sí; son los indicadores que presentan la menor distancia a 0 y en este caso conciernen a los siguientes ítems: ( $v 29 p c 22 r^{\prime}$ y 'v40pc35r'), en las figuras 6 y 7 , estos reactivos se unen en la primera etapa con un coeficiente de conglomeración (CC) de $(C C=, 000)$ según historial de conglomeración. Le siguen los indicadores ('v2 I pcl Ir' y 'v30pc23r') números (2 y 3) $(\mathrm{CC}=, 000)$ conglomerando en la segunda etapa. Estos cuatro indicadores constituyen el primer conglomerado, como se mencionó anteriormente, por estar al mismo nivel, por su cercanía natural y por su proximidad a cero. Al analizar en detalle el significado semántico de los dos primeros ítems mencionados, se comprueba la cercanía existente entre ellos por el contenido que estos profesan. Es decir, el primer ítem ('v29pc22r') se refiere a la relación de coherencia entre contenidos, metodología y evaluación, el otro indicador ('v40pc35r') hace mención a la relación 
Cuadro 2. Resultados de análisis de confiabilidad por componentes según criterios de relevancia y de susceptibilidad de mejora.

\begin{tabular}{|c|c|c|}
\hline Componentes & $\begin{array}{c}\text { Alfa de Cronbach } \\
\text { Relevancia }\end{array}$ & $\begin{array}{c}\text { Alfa de Cronbach } \\
\text { Susceptibilidad } \\
\text { Mejora }\end{array}$ \\
\hline DC & ,850 & ,927 \\
\hline$P C$ & ,900 & ,950 \\
\hline$P$ & ,667 & ,740 \\
\hline EC & ,667 & ,689 \\
\hline ENI & ,766 & ,799 \\
\hline C & ,920 & ,897 \\
\hline$A R$ & ,747 & ,683 \\
\hline TE & ,848 & ,848 \\
\hline INF & ,987 & ,768 \\
\hline$S$ & ,783 &, 716 \\
\hline ID & ,900 &, 953 \\
\hline \multicolumn{3}{|c|}{$\begin{array}{l}\text { Siglas: } \mathrm{DC}=\text { diseño curricular*, } \mathrm{PC}=\text { principios curriculares, } \mathrm{P}= \\
\text { perfil, } \mathrm{EC}=\text { estructura curricular, } \mathrm{ENI}=\text { elementos normativos } \\
\text { institucionales, } \mathrm{C}=\text { carrera*, } \mathrm{AR}=\text { autorregulación, } \mathrm{TE}= \\
\text { tutorías estudiantiles, INF= infraestructura, } \mathrm{S}=\text { seguimiento, } \\
\mathrm{ID}=\text { idoneidad docente. } * \text { Subdimensiones. }\end{array}$} \\
\hline
\end{tabular}

de congruencia entre la Escuela, la carrera y la institución. Ambos contenidos son semánticamente muy próximos, en el sentido de que determinan dos tipos de principios curriculares -coherencia y congruencia-, los cuales son importantes en el diseño curricular. Esta cercanía generada naturalmente por el Dendrograma apoya el significado de estos dos indicadores y su entorno de acción para lo que fueron creados en este estudio doctoral. Con respecto al segundo dúo de ítems, es decir ('v2 I pcl Ir' y 'v30pc23r'), el contenido del primero se refiere a la existencia de principios curriculares en el diseño del plan de estudios, y el otro indica la necesidad de que existan actividades analíticas que comprueben las relaciones de coherencia entre los elementos que conforman el perfil.Teniendo presente lo que indica la escala horizontal del Dendrograma, estos indicadores muestran una distancia euclídea menor a 5 unidades entre los centroides de los conglomerados; también se infiere que dichos indicadores quedan a una distancia re-escalada muy por debajo de 5, como se muestra en el gráfico del Dendrograma de Ward.

Un segundo conglomerado lo conforman los siguientes indicadores ('v120pc34r' y 'vl|9pc33r') números 12 y 13 del Dendrograma, $(C C=0,500)$, los que se agrupan en la tercera etapa según historial de conglomeración; le siguen los ítems (' $v \mid 2 p c 2 r$ ' y 'vl3pc3r), números 4 y 5 con un $(C C=, 1000)$

Dendrogram using Ward Method

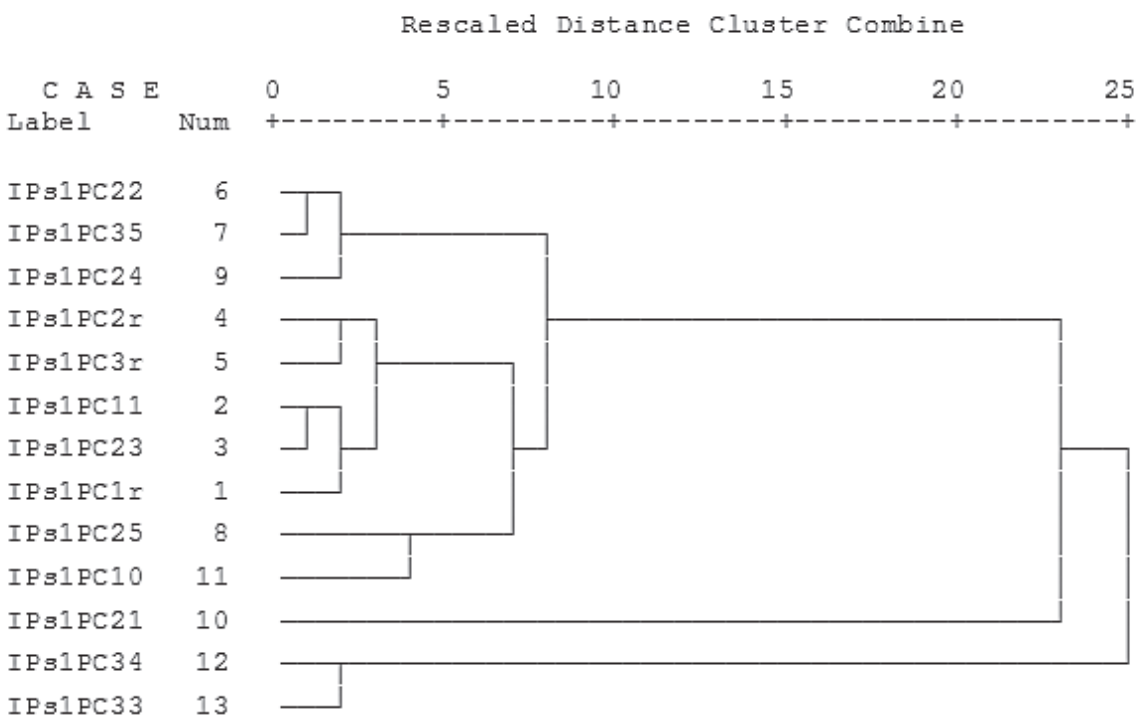

Figura 3. Dendrograma de Ward del componente Principios curriculares. 
congregándose en la cuarta etapa según historial de conglomeración; están también los reactivos ('v29pc22r'y'v3 I pc24r') números 6 y 9, (CC=1,667), los que se aglutinan en la quinta etapa según historial de conglomeración; se observan además (' $v \mid$ I pclr'y 'v2 I pc I Ir') números I y 2, (CC=2,333), agrupándose en la sexta etapa. Al examinar el resultado natural obtenido mediante el Dendrograma y desde el análisis del contenido que subyace en estos indicadores, se comprueba en el primer dúo de ítems ('vI20pc34r' y 'vl I9pc33r') la cercanía de ambos al expresar el primero ('v|20pc34r') la relación de congruencia entre cursos y formación; el segundo ítem ('v119pc33r') se refiere a la coherencia entre formación recibida y título otorgado. Con respecto a la segunda pareja (' $v \mid 2 p c 2 r$ ' y ' $v \mid 3 p c 3 r$ ), el contenido del primer indicador se refiere a la relación de coherencia entre Misión-Visión y objetivos institucionales y el segundo hace referencia a la necesidad de dejar bien explicito el objeto de estudio en el diseño curricular de la carrera. Estos mismos reactivos tiendan a conglomerarse relativamente pronto (tercera y cuarta etapa según historial). Teniendo presente lo que indica la escala horizontal del Dendrograma, estos indicadores muestran -al igual que el primer conglomeradouna distancia euclídea que no llega a las 5 unidades entre los centroides de los conglomerados, pues quedan -según nuestra apreciación- a una distancia re-escalada menor a 5, como se muestra en el gráfico del Dendrograma de Ward. Con respecto al tercer dúo, es decir, ('v29pc22r' y 'v3 I pc24r'), el contenido del primero se refiere a relación de coherencia entre contenidos, metodología y evaluación y el otro hace mención a la relación de coherencia entre el creditaje y el título. La cercanía de ambos queda confirmada al comprobarse que sus contenidos se refieren al principio curricular de coherencia, el cual como advertíamos antes es uno de los más importantes en materia de diseño curricular; por lo tanto la cercanía de ambos indicadores es más que evidente. Los contenidos de la última pareja de este grupo, es decir, los indicadores (' $v|| p c \mid r$ ' y ' $\mathrm{V} 2|p c| \mid r$ '), también se muestran relacionados al referirse a principios curriculares como el dúo anterior. El contenido del primero relata la relación de coherencia entre el plan de estudios y la profesión y el segundo la existencia de principios curriculares en el diseño del plan de estudios.

Un tercer conglomerado está formado por los indicadores ('vI2pc2r' y 'v30pc23r'). El contenido del primer ítem se refiere a la relación de coherencia entre la Misión-Visión y los objetivos institucionales y el segundo relata la necesidad de contar con actividades analíticas para la comprobación de la correspondencia entre contenidos y facultades cognitivas y actitudinales del plan de estudios. La cercanía de ambos queda comprobada según CC. El contenido de ambos se relaciona mediante el principio curricular de coherencia, el cual tiene gran importancia ya que extiende significativamente el sentido de correspondencia que debe existir entre los elementos microcurriculares (como la coherencia entre contenidos y competencias formativas) y los elementos macrocurriculares referentes a la orientación y a la razón de ser de una institución universitaria como el ITCR (visualizada mediante su Misión y Visión). La existencia de estas relaciones beneficia la formación integral de los estudiantes y la reputación de la institución.

El cuarto conglomerado lo conforman los indicadores ('v32pc25r" y 'v20pclOr'). El contenido del primero se refiere a la necesidad de contar con lineamientos curriculares para la interdisciplinariedad; el segundo describe la necesidad de contar con lineamientos pedagógicos en el diseño curricular. Ambos contenidos abogan por la urgencia de lineamientos, por lo cual su cercanía temática es clara tanto como la cercanía natural que se evidencia en el Dendrograma. El quinto conglomerado lo componen los indicadores ('v32pc25r' y ' $v \mid 3 p c 3 r$ '). El contenido del primero, como ya se indicó, se refiere a la necesidad de contar con lineamientos curriculares para la interdisciplinariedad; el otro, como también se indicó con anterioridad, se refiere a la necesidad y urgencia de contar en forma clara con el objeto de estudio en el diseño curricular de la carrera. La cercanía de ambos es evidente en el sentido de referirse a lineamientos curriculares que son importantes en el diseño de una profesión.

El sexto conglomerado está compuesto por los indicadores ('v40pc35r' y 'v30pc23r') cuyos contenidos también se refieren al principio curricular de coherencia. El primero hace alusión a la relación de congruencia entre la escuela, la carrera y la institución y el otro a la necesidad de contar con actividades analíticas para la comprobación de la correspondencia entre contenidos y facultades cognitivas y actitudinales del plan de estudios. Ambos contenidos son muy cercanos en la función que expresan del principio coherencia. 
El sétimo conglomerado lo forman los indicadores (' $v \mid 2 p c 2 r^{\prime}$ y ' $v|07 p c 2| r$ '). El contenido del primero se refiere a la relación de coherencia entre la MisiónVisión y los objetivos institucionales y el segundo relata la necesidad de la existencia de mecanismos que favorezcan la flexibilidad en el diseño curricular de las carreras de esta institución. Ambos contenidos se refieren a dos elementos fundamentales del currículo, es decir, la consideración de lo institucional y la presencia de mecanismos que favorezcan la flexibilidad en el diseño curricular. Estos dos componentes pertenecen al ámbito de lo macrocurricular y quizás es por este factor que se añade un poco de independencia a la relación de ambos en torno a los otros conglomerados y a la lejanía que se muestra en el Dendrograma. Sin embargo, la cercanía entre ambos es clara. El conglomerado más distante lo conforman los indicadores ('vI20pc34r' y 'v30pc23r'). El contenido de ambos está relacionado al referirse al principio de coherencia curricular. En ese sentido, el primero se refiere a la relación de coherencia entre cursos y plan de formación; el otro se refiere a la necesidad de incorporar actividades analíticas que comprueben las relaciones de coherencia entre los elementos que conforman el perfil. El primero presenta más variabilidad que el segundo. Asimismo, el rango promedio es bajo y presenta valoraciones atípicas en su evaluación. En relación con el contenido de ambos, se infiere que la lejanía que presentan podría estar relacionada con la ausencia de mecanismos que corroboren en forma precisa si los contenidos de los cursos están siendo coherentes, tanto con los elementos que conforman el perfil como con el plan de formación que los estudiantes actuales están necesitando. En el cuadro 3 se muestra el historial de conglomeración de este componente.

\section{Resultados del análisis de factores}

Los resultados obtenidos mediante la adecuación muestral o índice de $(\mathrm{KMO}=0,644)$, la prueba de esfericidad de Bartlett y la puntuación de significancia obtenida (Sig. $=, 000)$ son datos para proceder con la determinación de los factores en este análisis factorial. Se obtiene primero el análisis de "comunalidades" cuyos resultados se muestran en la cuadro 4, luego la matriz factorial y la varianza total explicada. Tal y como se observa en dicho cuadro, todos los puntajes obtenidos son mayores a $(0,70)$ superando con creces el valor tradicional de $(0,30)$. Dado que los resultados de las comunalidades son pesos factoriales bastante altos, se puede afirmar en este caso, basados en Hernández, Fernández y Baptista (2003, p. 57I), que, en efecto, "los ítems cargan o forman parte del factor o constructo correspondiente" y explican en una misma dirección el constructo al que representan. Se extrajo además la matriz de la varianza total explicada, la cual no se muestra por razones de espacio en este artículo. Se extrajo también la matriz factorial, la cual involucra el cálculo de los autovalores y la varianza total explicada. En este caso, tomando en cuenta las puntuaciones de las saturaciones, existen claramente tres factores mediante los cuales es posible explicar el 83,20\% de la varianza total explicada. El factor que más aporta a la explicación de la varianza es el I, que contribuye con un 53,58\%. Le sigue el factor 2 con un puntaje de $21,20 \%$ y el factor 3, que explica el $8,41 \%$ de la varianza. No obstante lo anterior, los dos primeros factores son las más significativos, ya que solo estos dos elementos explican el $74,79 \%$ de la varianza. El diagrama de sedimentación muestra la representación gráfica de la magnitud de los autovalores, los cuales, como se mencionó, son altos y han quedado bien definidos mediante el corte de inflexión de la cresta descendiente de dicho gráfico con los tres factores que mejor explican el total de la varianza explicada. Mediante la matriz de estructura factorial se obtuvo la solución factorial propiamente, la que contiene las correlaciones entre las variables originales o saturaciones y cada uno de los tres factores. Se aprecia que el primer factor está constituido por las variables referentes a la relevancia, todas las cuales saturan en un único factor porque constituyen un grupo diferenciado de variables dentro de la matriz de correlaciones. El segundo factor contiene las variables referentes a la susceptibilidad de mejora. El tercer factor está formado por una única variable que aparece no estar saturando en los otros dos factores, esta corresponde a PA_suscep. En la figura 5 se nota que dicha variable es la única que queda en el espacio tridimensional del componente del factor 3. Se muestran los Gráficos de Sedimentación y el de Componentes Rotados, figuras 4 y 5 , respectivamente.

Resultados obtenidos en torno al análisis de contenido de los indicadores de proceso de la dimensión Proceso curricular

Llegados a este punto, el fin primordial del análisis realizado es determinar en forma sintética categorías conceptuales con relación al contenido de los 
Cuadro 3. Historial de conglomeración del componente "principios curriculares"

\begin{tabular}{|c|c|c|c|c|c|c|}
\hline \multirow[t]{2}{*}{ Etapa } & \multicolumn{2}{|c|}{ Conglomerado que se combina } & \multirow{2}{*}{$\begin{array}{c}\text { Coeficientes } \\
\text { Conglomerado I }\end{array}$} & \multicolumn{2}{|c|}{$\begin{array}{c}\text { Etapa en la que el conglomerado } \\
\text { aparece por primera vez }\end{array}$} & \multirow{2}{*}{$\begin{array}{l}\text { Próxima etapa } \\
\text { Conglomerado } 2\end{array}$} \\
\hline & $\begin{array}{c}\text { Conglomerado } \\
\text { । }\end{array}$ & $\begin{array}{c}\text { Conglomerado } \\
2\end{array}$ & & $\begin{array}{c}\text { Conglomerado } \\
2\end{array}$ & $\begin{array}{c}\text { Conglomerado } \\
\mid\end{array}$ & \\
\hline । & 6 & 7 &, 000 & 0 & 0 & 5 \\
\hline 2 & 2 & 3 &, 000 & 0 & 0 & 6 \\
\hline 3 & 12 & 13 &, 500 & 0 & 0 & 12 \\
\hline 4 & 4 & 5 & 1,000 & 0 & 0 & 7 \\
\hline 5 & 6 & 9 & 1,667 & । & 0 & 10 \\
\hline 6 & 1 & 2 & 2,333 & 0 & 2 & 7 \\
\hline 7 & I & 4 & 3,567 & 6 & 4 & 9 \\
\hline 8 & 8 & 11 & 5,067 & 0 & 0 & 9 \\
\hline 9 & 1 & 8 & 8,024 & 7 & 8 & 10 \\
\hline 10 & I & 6 & 11,200 & 9 & 5 & 11 \\
\hline 11 & 1 & 10 & 20,864 & 10 & 0 & 12 \\
\hline 12 & 1 & 12 & 31,692 & 11 & 3 & 0 \\
\hline
\end{tabular}

Cuadro 4. Índices de comunalidades por dimensiones

Según análisis de factores

\begin{tabular}{|c|c|c|}
\hline & Inicial & Extracción \\
\hline PCurri_relevancia & 1,000 &, 748 \\
\hline Enseñanza_relevancia & 1,000 &, 831 \\
\hline PA_relevancia & 1,000 &, 783 \\
\hline PE_relevancia & 1,000 &, 853 \\
\hline Plrelevancia & 1,000 &, 881 \\
\hline PF_relevancia & 1,000 &, 807 \\
\hline PCurri_suscep & 1,000 &, 831 \\
\hline Enseñanza_suscep & 1,000 &, 847 \\
\hline PA_suscep & 1,000 &, 955 \\
\hline Plsuscep & 1,000 &, 786 \\
\hline PF_suscep & 1,000 &, 787 \\
\hline PE_suscep & 1,000 &, 874 \\
\hline
\end{tabular}

Método de extracción:Análisis de Componentes principales. indicadores que han sido evaluados como a.- los más relevantes y más susceptibles de mejora, b.- los menos relevantes y los menos susceptibles de mejora, c.- los que han sido evaluados tanto relevantes como susceptibles de mejora. Para la categorización indicada, se tomaron como base los resultados obtenidos en los análisis de Media aritmética y de Rangos promedio así como también la clasificación de ítem por disposición de la relevancia y de la susceptibilidad de mejora según perfil de importancia que se realizó. Mediante las figuras 6 y 7 se muestran las Medias logradas y el cuadro 5 despliega la clasificación de indicadores por disposición de la relevancia y de la susceptibilidad de mejora.

\section{Conclusiones}

Los resultados de validez alcanzados en esta investigación ofrecen evidencia suficiente para respaldar los indicadores de proceso como ítems de calidad, ampliamente válidos y confiables para proporcionar información válida, lícita, efectiva y confiable en la evaluación de la dimensión Proceso curricular. Con estos análisis, tanto el evaluador como las audiencias podrán tener la confianza y respaldo empírico 


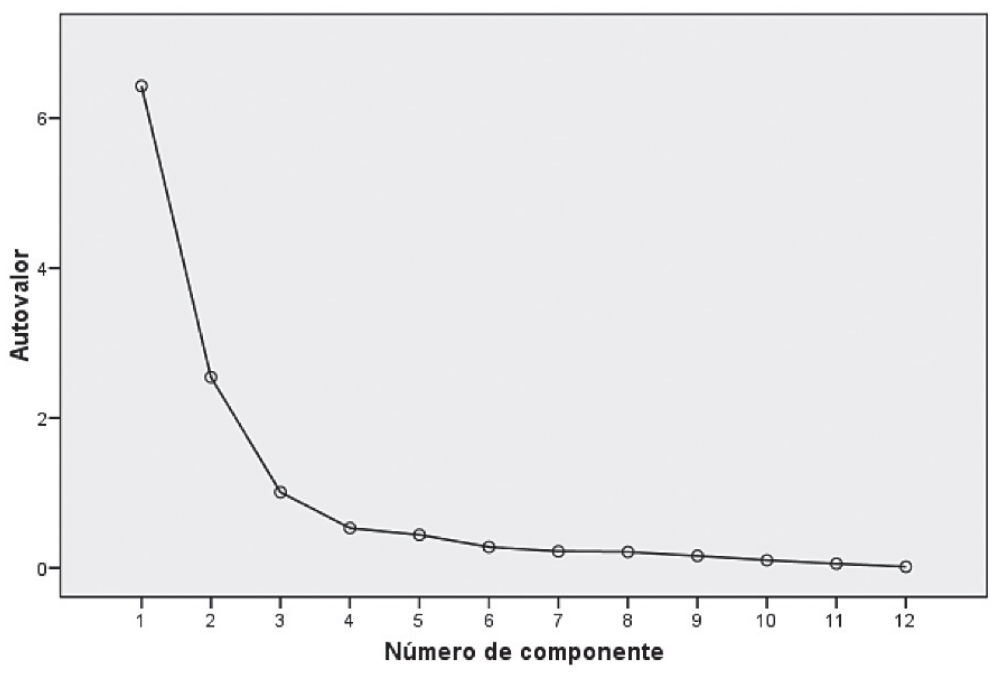

Figura 4. Gráfico de sedimentación por autovalores según número de componentes. Análisis de factores.

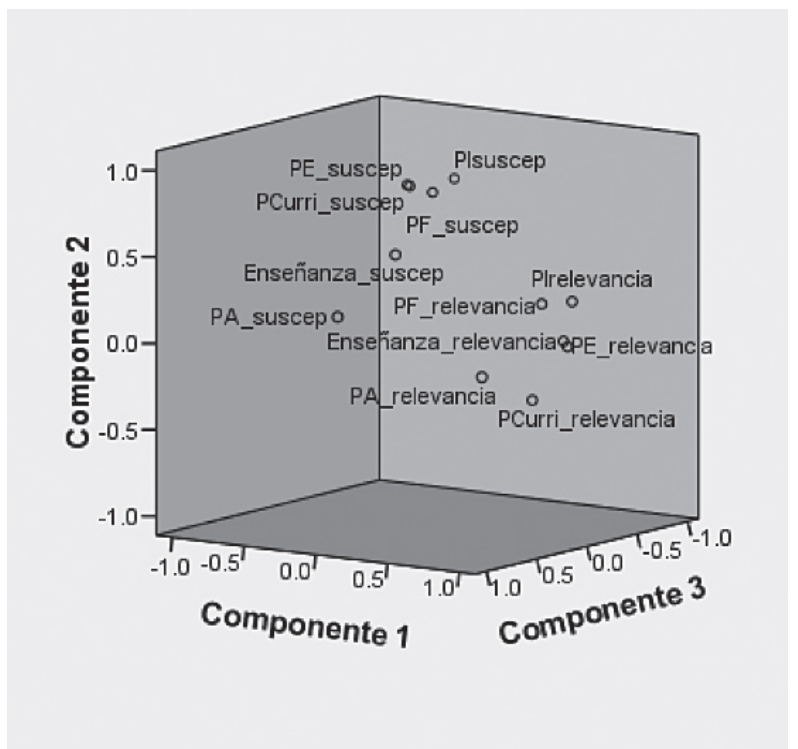

Figura 5. Componentes en espacio rotado según análisis de factores.

suficientes para aplicarlos en un proceso de evaluación y posteriormente en la toma de decisiones. Asimismo, podrán proveer y generar información pertinente sobre el estado actual de una de las dimensiones que constituyen el área de Formación universitaria. En ese sentido, los tres tipos de validez a los que fueron sometidos los indicadores de proceso, así como los resultados positivos logrados, son elementos garantes de la calidad técnica de estos ítems. Por consiguiente, se logra así alcanzar los objetivos que enmarcan este escrito.
Teniendo en cuenta lo señalado en páginas anteriores por Krippendorff (1997, p. 8I) y lo que anotan Hernández, Fernández y Baptista (2003, p. 347) en torno a la validez de contenido, se concluye que ésta quedó determinada mediante el análisis Perfil de importancia respecto al contenido de los indicadores, así como también por el análisis de media aritmética, de rangos promedio y del cociente de variación, como ya se mencionó. Estos cuatro tipos de análisis hicieron posible identificar categorías conceptuales en relación intrínseca con los contenidos de los enunciados de los indicadores valorados con la media y rangos más altos de cada componente. En consecuencia, al triangular estos hallazgos con los análisis de conglomerados jerárquicos se logra demostrar que las agrupaciones naturales y matemáticas -que genera dicho análisis a través del método Ward- son congruentes con las categorías conceptuales encontradas mediante el análisis de Perfil de importancia. Por tanto, lo anterior significa que los indicadores construidos, y que tienen como fin valorar la pertinencia y la susceptibilidad de mejora de la dimensión Proceso curricular, son ítems que "reflejan el dominio específico del contenido del concepto medido" para lo que fueron construidos y mediante sus "componentes semánticos facilitan las inferencias válidas acerca de los datos reunidos", tal y como afirman Hernández, Fernández y Baptista (2003, p. 347) y Krippendorff (1997, p. 8I), respectivamente, en sus correspondientes definiciones de validez de contenido, ya anotadas con anterioridad en el punto 3 de este artículo. Por consiguiente, los 


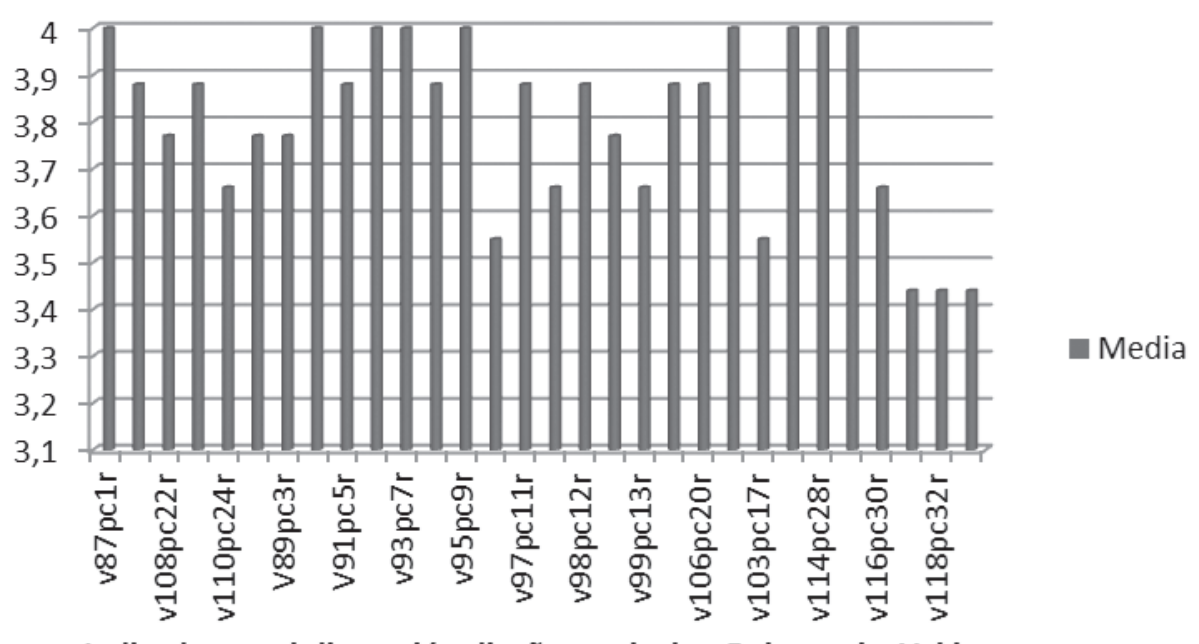

Indicadores subdimensión diseño curricular. Relevancia. Val interna

Figura 6. Media de relevancia. Indicadores subdimensión diseño curricular.Validación interna.

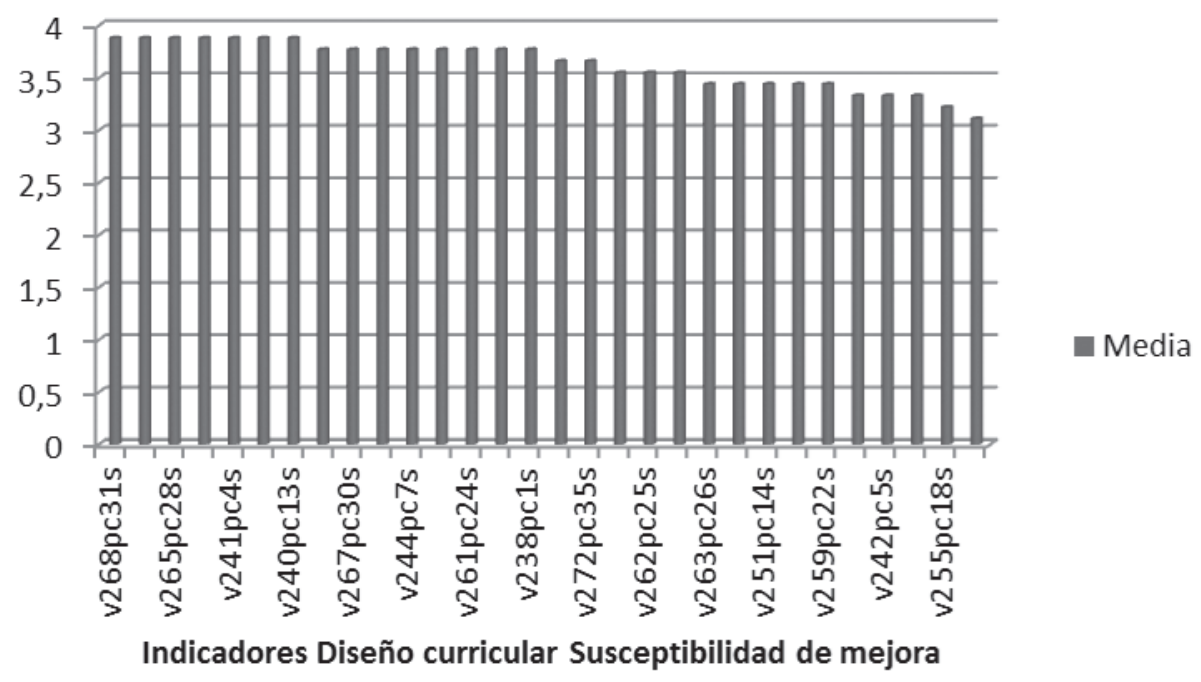

Figura 7. Media de susceptibilidad de mejora. Indicadores subdimensión diseño curricular.Validación interna.

indicadores de proceso aquí analizados tienen una validez de contenido totalmente comprobada.

De igual manera, con relación a la validez de constructo y haciendo alusión a los dos análisis (conglomerados jerárquicos y análisis de factores) utilizados para demostrar dicha validez, se concluye que: En relación directa con el primero: este se utilizó con el fin de establecer grupos o tipologías semánticas entre los indicadores creados, a fin de descubrir agrupaciones naturales entre ellos, lo más homogéneas a lo interno de éstas. Lo anterior se logró mediante el Dendrograma de Ward, aplicando el método Ward como se indicó en el apartado de resultados. Con este análisis se demostró que la cercanía natural de los indicadores está conforme con las tipologías semánticas que respaldan el enunciado presente en cada uno de los ítems, corroborando de esta manera la validez de constructo de dichos indicadores.

Entrando en mayores precisiones, siempre en la línea del análisis de conglomerados jerárquicos, validez de constructo, pero específicamente en torno al 
Cuadro 5. Clasificación de ítems por disposición de relevancia y de susceptibilidad de mejora según análisis Perfil de importancia respecto al contenido de los indicadores de la Dimensión Proceso curricular.Validación externa.

\begin{tabular}{|c|c|c|c|c|c|}
\hline \multicolumn{6}{|c|}{ DIMENSIÓN PROCESO CURRICULAR } \\
\hline \multicolumn{3}{|c|}{ Subdimensión Diseño Curricular } & \multicolumn{3}{|c|}{ Subdimensión Carrera } \\
\hline Indicador DC & Media relevancia DC & $\begin{array}{c}\text { Media Suscep } \\
\text { DC }\end{array}$ & Indicador Carrera & $\begin{array}{c}\text { Media Relevancia } \\
\text { Carrera }\end{array}$ & $\begin{array}{c}\text { Media Suscep M } \\
\text { Carrera }\end{array}$ \\
\hline v18pc8r & 4,00 & 3,82 & $v 46 p c 44 r$ & 4,00 & 3,52 \\
\hline v16pc6r & 4,00 & 3,76 & v60pc63r & 3,94 & 3,64 \\
\hline$v|| p c \mid r$ & 3,94 & 3,76 & v48pc46r & 3,94 & 3,94 \\
\hline v22pc|2r & 3,88 & 3,88 & v47pc45r & 3,94 & 3,41 \\
\hline v29pc22r & 3,88 & 3,76 & v42pc37r & 3,94 & 3,58 \\
\hline$v \mid 2 p c 2 r$ & 3,88 & 3,76 & $v 4 \mid p c 36 r$ & 3,88 & 3,64 \\
\hline$v 24 p c \mid 4 r$ & 3,82 & 3,70 & v62pc65r & 3,88 & 3,52 \\
\hline$v 23 p c \mid 3 r$ & 3,82 & 3,64 & v54pc54r & 3,88 & 3,52 \\
\hline$v 17 p c 7 r$ & 3,82 & 3,47 & $v 45 p c 42 r$ & 3,88 & 3,82 \\
\hline v31pc24r & 3,82 & 3,76 & $v 56 p c 56 r$ & 3,82 & 3,41 \\
\hline$v 25 p c 16 r$ & 3,76 & 3,52 & v44pc40r & 3,82 & 3,76 \\
\hline v40pc35r & 3,70 & 3,76 & $v 52 p c 5 \mid r$ & 3,76 & 3,76 \\
\hline v35pc $28 r$ & 3,70 & 3,70 & v58pc60r & 3,76 & 3,52 \\
\hline v34pc27r & 3,70 & 3,76 & v57pc57r & 3,76 & 3,64 \\
\hline v32pc25r & 3,70 & 3,76 & v55pc55r & 3,76 & 3,52 \\
\hline v27pc|8r & 3,70 & 3,47 & v5 I pc50r & 3,76 & 3,58 \\
\hline v33pc26r & 3,70 & 3,70 & v50pc48r & 3,64 & 3,58 \\
\hline v26pc|7r & 3,70 & 3,52 & v65pc69r & 3,58 & 3,41 \\
\hline v19pc9r & 3,64 & 3,52 & v63pc66r & 3,58 & 3,35 \\
\hline v37pc30r & 3,64 & 3,76 & v6 I pc64r & 3,58 & 3,35 \\
\hline v30pc23r & 3,64 & 3,58 & $\vee 53 p c 52 r$ & 3,52 & 3,70 \\
\hline v36pc29r & 3,58 & 3,70 & v43pc38r & 3,47 & 3,52 \\
\hline$v 13 p c 3 r$ & 3,58 & 3,52 & v59pc6 Ir & 3,47 & 3,35 \\
\hline v39pc32r & 3,52 & 3,70 & v49pc47r & 3,41 & 3,88 \\
\hline v38pc3Ir & 3,47 & 3,70 & v64pc68r & 2,94 & 3,17 \\
\hline v। $5 p c 5 r$ & 3,41 & 3,70 & & & \\
\hline$v \mid 4 p c 4 r$ & 3,41 & 3,64 & & & \\
\hline v20pclor & 3,23 & 3,41 & & & \\
\hline$v 2|p c| \mid r$ & 3,23 & 3,47 & & & \\
\hline v28pc20r & 3,00 & 3,23 & & & \\
\hline \multicolumn{6}{|c|}{$\begin{array}{l}\text { Código: } \\
\text { Ítems con fondo gris claro= Indicadores más relevantes y más susceptibles de mejora. } \\
\text { Ítems con fondo en blanco= Indicadores que guardan cierta similitud entre el criterio de relevancia y de susceptibilidad de mejora. } \\
\text { Ítems con fondo gris oscuro= Indicadores menos relevantes y menos susceptibles de mejora. } \\
\text { DC= diseño curricular. Suscep= susceptibilidad de mejora. }\end{array}$} \\
\hline
\end{tabular}


área curricular para la cual fueron construidos los indicadores de proceso, se concluye lo siguiente en relación con la subdimensión Diseño Curricular, la cual "es el componente vital del engranaje que forma el proceso curricular" (Lira, 20 I I, p. 23). En dicha área, en el componente Principios curriculares, el análisis de conglomerados jerárquicos pone a la vista la conglomeración casi inmediata de cuatro indicadores (v29pc22r' y 'v40pc35r') y ('v2 I pc I I r' y 'v30pc23r'), (primera y segunda etapa con coeficiente de conglomeración $C C=000$ ) primer conglomerado como se mencionó en el punto 5.3. Producto de esta agrupación y de la categorización realizada, se evidencian los principios de coherencia y congruencia como pilares indispensables para la conformación de diseños curriculares funcionales. Lo importante de esta agrupación matemática obtenida es poder concluir y demostrar, especialmente a los escépticos del currículo, que la cercanía generada naturalmente por el Dendrograma sustenta el significado de esos indicadores y su entorno de acción en la creación de carreras universitarias y sus procesos formativos. Se concluye, por tanto, que en materia de diseño curricular es indispensable contar con coherencia entre perfil, contenidos, metodología y evaluación para generar enseñanza y aprendizajes efectivos. Asimismo, el hecho de que exista congruencia entre los fines de la institución, la escuela y la carrera, genera armonía en los fines formativos que pretende la universidad. Al juntar los contenidos de estos cuatro componentes del primer conglomerado se comprueba que, en efecto, estos indicadores conforman un conjunto de elementos cuya semántica queda referida a elementos normativos insustituibles para el diseño curricular de las carreras universitarias. Por lo cual, es satisfactorio comprobar mediante el Dendrograma la unicidad existente entre ellos, la cual concuerda con el contenido que profesan dichos indicadores en ese primer conglomerado.

Igualmente, al juntar los ítems que conforman el segundo conglomerado (' $v 120 p c 34 r^{\prime}$ y ' $v 119 p c 33 r$ '), ('vI2pc2r' y 'vI3pc3r, ('v29pc22r' y 'v3lpc24r'), (' $v|| p c \mid r$ ' y ' $v 2|p c| \mid r$ ') y teniendo en cuenta las categorías semánticas resultantes del contenido que los embarga, se concluye que las categorías semánticas de estos ítems son mutuamente interdependientes. Por tanto, es indispensable que se mantenga esa simbiosis de contenido para que exista unicidad entre la formación y la certificación que otorga la universidad como compromiso asumi- do con la sociedad civil. De ahí que es importante la cercanía natural de estos dúos, la cual se comprueba por efecto de conglomeración jerárquica. Se concluye con respecto a la aglutinación de la segunda pareja de ítems ('vI2pc2r' y 'vI3pc3r) que la proximidad natural de estos indicadores evidenciada por el Dendrograma resulta importante, ya que la semántica de dichos reactivos se refiere a aspectos del diseño curricular que llevan a generalizaciones más amplias como las siguientes: primero, las relaciones de coherencia entre la misión/visión/ objetivos institucionales (' $v \mid 2 p c 2 r$ ') hacen que el diseño curricular de las profesiones trascienda la organización; segundo, que el diseño curricular sea considerado un proceso de construcción social y no solamente como un simple acto de acomodamiento de cursos académicos. El hecho de que el ítem ('vl3pc3r) señale que el objeto de estudio de la carrera está bien claro y sea evidente en el diseño curricular implica darle a este elemento el lugar de importancia que le corresponde. El elemento "objeto de estudio" es el referente por excelencia para caracterizar una profesión (Horruitiner, 2006, p. 8) y determinar las áreas de desempeño laboral hacia las que se dirige el plan formativo. Es, además, el encargado de delimitar las competencias que adquirirá el estudiante en el periodo de su formación. Es satisfactorio, para los efectos de la investigación, poder comprobar la unicidad natural de acople que presentan estos indicadores (' $v \mid 2 p c 2 r^{\prime} y^{\prime} \mathrm{v} I 3 p c 3 r$ ) y que son contundentemente evidentes mediante el Dendrograma de Ward.

Del análisis realizado a los nueve componentes que conforman la dimensión Proceso curricular se concluye en forma fehaciente que los datos obtenidos demuestran que existe cercanía natural y uniformidad consistente entre los indicadores de los diferentes componentes a los que pertenecen. Se manifiesta que existe cercanía a cero en bastantes conglomerados, pues la mayoría de ellos queda a una distancia re-escalada de 5 del resto, tal y como se demostró en el dendrograma que se generó en forma independiente para cada componente. Este dato corrobora la validez de constructo de los indicadores que conforman esta dimensión. Asimismo, estos datos confirman la importancia de los enunciados que poseen los indicadores para el área curricular y en particular para el Proceso Curricular y sus componentes. 
En relación con el análisis de factores, en forma general y en referencia específica a dos de las cuatro partes que constituyen el análisis factorial, es decir, a la solución factorial y a la composición de cada factor, se concluye lo siguiente:

A. Con respecto a la estructura general de la solución factorial, esta quedó constituida por tres factores, principalmente después de la rotación con cargas factoriales mejor distribuidas que antes de la rotación. Dichas cargas factoriales que conforman estos tres componentes puntúan alto y son positivas, oscilando entre $(0,783$ y 0,922$)$. Ninguna de estas es bipolar, por lo que están representando el respectivo constructo en forma significativa.

B. Con respecto a la composición de cada factor (e involucrando las otras dimensiones y el otro tipo de indicadores construidos para efectos de la propuesta madre), se afirma que el componente o factor I queda conformado por 6 variables homogéneas que conforman las dimensiones: PCurri_relevancia, Enseñanza_relevancia, PA_relevancia, PE_relevancia, PI_relevancia y PF_relevancia, en las cuales se encuentran los indicadores de proceso y de producto que han sido creados para medir la relevancia de las áreas de Formación universitaria y de Impacto. Sus respectivas cargas factoriales unipolares son, en el mismo orden de mención, las siguientes: (,820), (,910), (,783), (,922), (,898) y $(, 965)$, todas las cuales saturan en el mismo factor porque constituyen un grupo diferenciado dentro de la matriz de correlaciones y reafirmada al aplicar la rotación: Normalización Oblimin con Kaiser. El factor 2 queda constituido por 5 variables también homogéneas que instituyen las dimensiones de: Enseñanza_suscep, Pl_suscep, PF_suscep y PE_suscep, en las que se agrupan los indicadores de proceso y de producto que han sido elaborados precisamente para medir la posibilidad o susceptibilidad de mejora que tienen dentro de las áreas de Formación Universitaria y de Impacto que conforman la propuesta modélica madre, de la cual este artículo es una parte, como se indicó al inicio. Las cargas factoriales correspondientes que saturan en este factor de estas variables son: $(, 736),(, 874),(, 881)$ y $(, 922)$ en orden respectivo. El factor 3 está conformado por una única variable que comprende la dimensión PA_suscep constituida por indicadores de proceso cuyo peso factorial es alto (,95I) y es unipolar. Los tres factores logrados son independientes entre sí, ya que saturan en uno solo de los factores. Por todo lo anteriormente expuesto, se considera que los resultados obtenidos son satisfactorios y mediante ellos se ha comprobado la validez de constructo de las dimensiones e indicadores de proceso que conforman el área de Formación Universitaria en la cual se ubica la dimensión Proceso curricular con sus respectivos componentes.

En relación con la confiabilidad y según los resultados obtenidos mediante las puntuaciones del Alfa de Cronbach, se comprueba que dichos valores son significativos para los efectos de esta investigación. Así se desprende del análisis realizado a los diez componentes que conforman la dimensión Proceso Curricular y que fueron mostrados en el cuadro 2. Se concluye que todos presentan valores aptos, altos y positivos de confiabilidad. Dichos valores señalan que los indicadores que representan cada componente no muestran ninguna ambivalencia en sus enunciados o contenidos; señalan además un uso confiable, adecuado y consistente de los indicadores que conforman cada uno de los componentes y sus respectivas dimensiones. El análisis por componentes también indica que los resultados de fiabilidad son altos y positivos para los efectos para los que han sido creados.

Finalmente, y de forma general, se concluye que los indicadores de proceso construidos son representantes relevantes y susceptibles de mejora de los componentes a los que pertenecen; denota además el enunciado que cada indicador posee una condición viable cuya condición de mejora o susceptibilidad de mejora se puede obtener. Los resultados obtenidos en estos análisis son positivos y permiten proponer indicadores de calidad que contienen validez de constructo comprobada para la evaluación de la dimensión Proceso Curricular en la Educación Superior. Estos constituirán un insumo más para ser considerado por evaluadores y por audiencias específicas para la evaluación de esta importante área de la formación universitaria y servirán de referentes indispensables para la toma de decisiones en términos de mejora institucional. 


\section{Bibliografía}

Alvira Martin, F. (1985). La investigación evaluativa: una perspectiva experimentalista. Revista Española de Investigaciones Sociológicas Enero-Marzo, (29): |29-|4|.

Bodgan, R.C. \& Binklen, S.K. (1982). Qualitative Research for Education: an Introduction to Theory and Methods. Boston: Allyn \& Bacon.

Carr, Wilfred \& Kemmis, S. (1988). Teoría crítica de la enseñanza. La investigación acción en la formación del profesorado. Barcelona: Ediciones Martínez Roca.

Coll, C. (1998). Pedagogía y Curriculum. Barcelona: Editorial Laia S.A.

Cook, T.D. \& Reichardt, CH. (1997). Métodos cualitativos y cuantitativos en investigación evaluativa. España: Morata.

De Alba, A. (1994). Curriculum, crisis, mito y perspectivas. México: Universidad Nacional de México.

De la Torre, S. (1999). Creatividad en la reforma española. Revista de Educación (319): 187-198.

Días Barriga, Á. (1984). El curriculum de pedagogía: un estudio exploratorio desde una perspectiva estudiantil. UNAM, México: ENEP-Aragón.

Flórez Ochoa, R. (2000). Hacia una pedagogía de conocimiento. Santafé de Bogotá, Colombia: McGraw Hill Interamericana S.A

Follari, R. \& Berruezo, J. (198I). Criterios e instrumentos para la revisión de planes de estudio. Revista Latinoamericana de Estudios Educativos, XI ( I): | 6 | - |85.

Grundy, S. (I 897). Curriculum: Product or Praxis. US: Falmer Press.

Hernández, R., Fernández, C. \& Baptista, P. (2003). Metodología de la investigación. México: McGraw-Hill.

Horruitiner Silva, P. (2006). El reto de la transformación curricular. Revista Iberoamericana de Educación 4(3): I - 13.

Jornet, J.M \& Suárez, J.M. (2008b). Métodos de Investigación y Análisis de datos: Conceptos básicos introductorios generales. Material de clases del Doctorado en Intervención. Universidad de Valencia: Grupo GEM.
Krippendorff, K. (1997). Metodología de análisis de contenido: teoría y práctica. Barcelona: Paidós.

Lira Valdivia, R.I. (20l0). Normas y orientaciones para el diseño curricular en el Instituto Tecnológico de Costa Rica. Guía Metodológica. Documento inédito disponible en el Centro de Desarrollo Académico. Cartago, C.R.: CEDA/TEC.

Lira Valdivia, R.I. (20 I0). Las metodologías activas y el foro presencial: su contribución al desarrollo del pensamiento crítico. Actualidades Investigativas en Educación IO(I) | - I8.

Lira Valdivia, R.I. (20 I I). Fundamentos del Currículo: una visión para el CEDA-TEC. Documento inédito disponible en el Centro de Desarrollo Académico. Cartago, C.R.; CCEDA/TEC.

Lira Valdivia, R.I. (20 I 3). Diseño de un enfoque modélico de evaluación institucional para el Instituto Tecnológico de Costa Rica: Construcción y validación de los indicadores de proceso y de Producto de las áreas de formación universitaria y de impacto que forman dicho modelo. Tesis para optar por el grado de Doctora en Intervención Educativa, Universidad de Valencia, España.

McMillan, J. \& Schumacher, R. (2005). Investigación educativa. Madrid: Pearson.

Posner, G. (2005). Análisis del currículo. Santafé de Bogotá, Colombia: McGraw Hill Interamericana.

Rodríguez Jaume, M.J. (2000). Modelos sociodemográficos. Tesis para optar por el grado de Doctorado en Economía, Universidad de Alicante.

Ruthman, L. (1977). Evaluation Research Methods: a Basic Guide. London: Sage.

Stenhouse, L. (199|). Investigación y desarrollo del curriculum. España: Morata.

Torres Acuña, W.J. (2003). Utilización de internet y el bienestar psicológico en estudiantes universitarios de alto y bajo nivel de acceso a la tecnología por computadora. Tesis para optar por el grado académico de Doctorado en Psicología, Universidad Nacional Mayor de San Marcos, Lima. Obtenido el 14 de septiembre de 2012 desde http://sisbib.unmsm.edu.pe/bibvirtualdata/Tesis/Salud/torres_aw/T_completo.pdf 